\title{
Management of Subtrochanteric Fracture Femur by Proximal Femoral Nail
}

\author{
Dr. Gurluv Singh Jaura ${ }^{1}$, Dr. Gurinder Gosal ${ }^{2}$ \\ ${ }^{1}$ Senior Resident, G.G.S. Medical College \& Hospital, Faridkot - 151203, Punjab, India
}

\begin{abstract}
Background: Subtrochanteric fractures of the femur remain some of the most challenging fractures faced by orthopaedic surgeons. Most of the fractures in the elderly results from trivial fall from standing or while walking, whereas in the younger age group it is mainly due to road traffic accidents. Closed management of these subtrochanteric fractures poses difficulties in obtaining and maintaining reduction, hence making operative management the preferred method of treatment ${ }^{1}$.Rapid strides in new implants, instrumentation and fracture fixation techniques in quest for ideal implant for fixation of these fractures have made many options available. This study was conducted to evaluate the outcome of management of 60 adult patients with subtrochanteric femoral fractures using proximal femoral nail(PFN). Materials and Method: This prospective study included 60 patients with subtrochanteric fracture admitted to Guru Gobind Singh Medical College, Faridkot who underwent intramedullary fixation with PFN from May 2012 to August 2014. Only fresh subtrochanteric fractures within one week of injury were included in the study. Pathologic fractures, multiple fractures, fractures in children, old neglected fractures were excluded from the study. Results: In our study of 60 patients, there were 45 male and 15 female patients with mean age of 36 years( range from 17- 68 years).Sixty seven percent of the patients admitted were road traffic accidents, 23\% due to fall from height and $10 \%$ due to trivial fall with right side being more common side affected. Russell and Taylor type IA fracture accounted for $40 \%$ of cases. Mean duration of hospital stay was 7 days and mean time of full weight bearing was 14 weeks in our patients. Out of 60 patients, 3 patients were lost to follow up and 1 case died due to associated medical co-morbidity. Good to excellent results were seen in $80 \%$ of cases in our study. Conclusion: PFN is a viable treatment option for subtrochanteric fractures with high rate of fracture union and minimal soft tissue damage. Intramedullary fixation has biological and biomechanical advantages, but the operation is technically demanding. Gradual learning and great patience is needed in order to make this method truly minimally invasive.
\end{abstract}

Keywords: PFN, Subtrochanteric fractures, Russell and Taylor.

\section{Introduction}

Subtrochanteric fractures of femur include fractures that occur below the lesser trochanter to $5 \mathrm{~cm}$ distally in the shaft of femur ${ }^{2,3}$. These fractures occur typically at the junction between trabecular bone and cortical bone where the mechanical stress across the junction is highest in the femur, which is responsible for their frequent comminution. These fractures occur typically in two age groups. In young and healthy individuals, the injury results from high-energy trauma, whereas in the elderly population, most of the fractures are due to osteoporotic bones, resulting from a fall. With the increase in the aging population, there is also considerable growth in the number of pathological fractures and fractures around hip prostheses (periprosthetic fractures).The most appropriate implant for internal fixation of subtrochanteric fractures remains a subject of debate, and many different intramedullary and extramedullary implants have been advocated for surgical fixation of these fractures ${ }^{4}$.Earlier the solution for such fractures was open reduction and internal fixation of these fractures but this method of treatment has changed substantially over the past decades. Earlier anatomical reduction and rigid internal fixation was the treatment of choice which required too much soft tissue dissection and muscle dissection leading to the fragment becoming avascular. These disadvantages have been overcome by intramedullary nailing which has the advantage of insertion using closed technique, retaining the fracture haematoma, lesser soft tissue dissection and thus less infection rate. Closed nailing constitues a form of biological fixation of the femur which may be credited for a shorter time to union. The two primary options for treatment of subtrochanteric fractures are intramedullary fixation and extra medullary fixation ${ }^{5}$.Many internal fixation devices have been recommended, but because of high incidence of complications like non-union and implant failure, a series of evolution in designing a perfect implant has begun. Only recently better understanding of biology, reduction techniques and biomechanically improved implants like Gamma nail, Russell Taylor nail, Proximal femoral nail allowed for these fractures to be addressed with consistent success. This study was conducted to determine the rate of union, complications, operative risks and functional outcomes in subtrochanteric fractures treated with proximal femoral nail.

\section{Materials and Methods}

This prospective study included 60 adult patients with subtrochanteric fractures of femur who were treated with PFN in Guru Gobind Singh Medical College, Faridkot from May 2012 to August 2014.The fractures were classified according to Russell and Taylor classification. Fifty six patients were followed at regular intervals and 3 were lost to follow up and 1 patient died due to associated medical comorbidity. Only fresh subtrochanteric fractures within one week of injury were included in the study. Pathological fractures, fractures in children, old neglected fractures and peri prosthetic fractures were excluded from this study. All these 60 patients underwent fracture reduction and internal fixation of subtrochanteric fracture using PFN (Synthes AO).The patient was placed in supine position on fracture table with adduction of the affected limb by 10-15 degrees and closed reduction of the fracture was done by the traction and internal rotation. The unaffected leg was flexed and abducted as far as possible or kept in wide abduction. The image intensifier was positioned so that anterior-posterior and lateral views of hip and femur could be taken. Open reduction was performed if closed 


\section{International Journal of Science and Research (IJSR) \\ ISSN (Online): 2319-7064}

Index Copernicus Value (2013): 6.14 | Impact Factor (2014): 5.611

reduction failed. A skin incision measuring $3-5 \mathrm{~cm}$ was made proximal to tip of greater trochanter on the proximal extension of anatomical femoral bow. Skin ,subcutaneous tissue and fat separated and gluteal muscle split along its fibres. Tip of greater tronchanter was exposed. In AP view under image intensifier, the entry point was selected as tip of greater tronchanter. In lateral view, guide wire position was confirmed in the center of the medullary cavity. The medullary canal was entered with a curved bone awl, the guide wire was inserted into the medullary canal. Using a cannulated conical reamer proximal femur was reamed for a distance of about $7 \mathrm{cms}$. After confirming satisfactory fracture reduction, an appropriate size nail as determined preoperatively and intraoperatively was assembled to insertion handle and inserted manually. This step was done carefully without hammering by slight twisting movements of the hand until the hole for $8 \mathrm{~mm}$ screw was at the level of inferior margin of the neck. Open reduction was performed in case satisfactory reduction was not possible by closed means. Guide wire for hip pin and neck screw were inserted with the help of aiming device lightly screwed to the insertion handle. A $2.8 \mathrm{~mm}$ guide wire was inserted through the drill sleeve after a stab incision. This guide wire was inserted $5 \mathrm{~mm}$ deeper than the planned screw size. The final position of the guide wire should be in the lower half of the neck in AP view and in the center of the neck in lateral view. A second $2.8 \mathrm{~mm}$ guide wire was inserted through the drill sleeve above the first one for hip pin. The tip of this guide wire was positioned approximately $25-20 \mathrm{~mm}$ less deep than planned neck screw. Drilling was done over $2.8 \mathrm{~mm}$ guide wire until the drill was $8 \mathrm{~mm}$ short of tip of the guide wire. Tapping was not done as neck screw is self tapping. Neck screw was inserted using cannulated screw driver. Similarly appropriate length hip pin was inserted. Length and position of the screw was confirmed under c-arm image intensifier. Distal locking was usually performed with two cortical screws. A drill sleeve system was inserted through a stab incision. A drill hole was made with $4 \mathrm{~mm}$ drill bit through both cortices. Locking screw was inserted and position was confirmed with image intensifier. After fixation was over, lavage was given using normal saline and incision was closed in layers. Suction drain was used in case open reduction was performed. Postoperatively, patients were encouraged to sit in the bed after 24 hrs following surgery. Patients were taught Quadriceps static exercise and knee mobilization in immediate postoperative period. Patients were taught gait training before discharge from hospital.All the patients were followed up at 4 weeks, 12 weeks and then at every 6 weeks interval thereafter till fracture union was noted.Patients were serially followed up at at 6 months, 9 months and 1 year. At each visit, patient was assessed clinically regarding hip and knee function, walking ability,fracture union, deformity and shortening. Hip function in each patient was assessed by using the Harris Hip Scoring System.

\section{Results}

Out of total 60 patients, 56 patients were available for follow up. Three patients were lost in follow up and 1 patient died due to associated medical comorbidity. Out of 56 patients, 52 patients achieved radiological union after a mean duration of 5.2 months(range 4.2-7.6 months)(Fig-1B and Fig-2B).In 4 patients fracture union was not achieved even after mean duration of 10.7 months. These 4 patients underwent exchange nailing and bone grafting, following which fracture union was achieved with mean duration of 4.8 months. Two out of 56 patients had malunion with average varus angulation of $12^{\circ}$. Two patients had shortening of average 1.5 $\mathrm{cm}$ which may be contributed to excessive comminution at the fracture site in these 2 patients. Two patients had superficial wound infection which required intravenous antibiotics for 3 weeks period. Z-effect was noted in 1 patient for which implant removal was done as fracture had already united. Reverse Z-effect was noted in one case for which neck screw removal was done as fracture had already united.

\section{Discussion}

Unlike osteoporotic trochanteric fractures, subtrochanteric fractures are usually the result of high energy trauma and often subjected to significant displacement and great difficulty in close reduction through traction. The high incidence of delayed union, malunion and non-union of fractures has left conservative treatment, as advocated by DeLee et al, abolished in modern trauma care ${ }^{6}$.Extra medullary fixation with plating has the potential disadvantages of extensive surgical exposure, severe soft tissue damage and blood loss, thus leading to problems of fracture union and implant failure. In addition, the eccentrically plating is prone to fatigue breakage due to their mechanical load-sharing effect.Allowing a minimally open approach, intramedullary nailing is closely linked to "biological internal fixation", in addition to its mechanical benefits over plate fixation. Intramedullary fixation allows the surgeon to minimize soft tissue dissection thereby reducing surgical trauma, blood loss, infection, and wound complications(Leung et al. 1992, Radford et al. 1993).A laboratory study (Mahomed et al. 1994) comparing a locked. Gamma nail to a standard sliding hip screw for the fixation of stable and unstable subtrochanteric fractures showed that the intramedullary nail was more rigid and permitted less fracture displacement and concluded that intramedullary fixation was superior to extramedullary fixation ${ }^{7}$.The currently used Gamma nail as an intramedullary device also has a high learning curve with technical and mechanical failure rates of about $10 \%$ (collapse of the fracture area, cutout of the implant, fracture of the femur shaft) ( Friedl 1996, Valverde et al. 1998). The Gamma nail is susceptible to fail at its weakest point, the lag screw implant interface. The AO ASIF in 1996, therefore developed the Proximal Femoral Nail to reduce the risk of implant related complications. Therefore in addition to the $8 \mathrm{~mm}$ load bearing femoral neck screw, the PFN has a $6.5 \mathrm{~mm}$ antirotation screw to increase the rotational stability of the neck fragment. An anatomic $6^{0}$ neck valgus bend in the coronal plane, a narrower distal diameter and distal flexibility of the nail eliminates the need for routine reaming of the femoral shaft and also minimizes stress concentration and tension in the femoral shaft. This should reduce the risk of intraoperative and postoperative femoral shaft fractures.PFN also has all the advantages of an intramedullary device such as decreasing the moment arm, can be inserted by closed technique which retains the fracture heamatoma, decreases blood loss, minimizes soft tissue dissection and wound infections. In an experimental

\section{Volume 4 Issue 11, November 2015}




\section{International Journal of Science and Research (IJSR) \\ ISSN (Online): 2319-7064}

Index Copernicus Value (2013): 6.14 | Impact Factor (2014): 5.611

study Gotze et al (1998) compared the loadability of osteosynthesis of unstable per and subtrochanteric fractures and found that the PFN could bear the highest loads of all devices.Since its introduction in 1997 several clinical studies have shown good result with few intra operative problems and low rates of complications ${ }^{8}$. Werner et al was the first who introduced the term Z-effect, detected in $5(7.1 \%)$ of 70 cases. The incidence of cut-out of the neck screw in this study was $1.6 \%$. The Z-effect phenomenon is referred as a characteristic sliding of the proximal screws to opposite directions during the postoperative weight-bearing period.In our study we had Z-effect in one case(1.6\%)(Fig-3A). The reverse Z-effect described by Boldin et al occurred with movement of the hip pin towards the lateral side, which required early removal ${ }^{9}$. The mechanism is similar, but here the hip pin is sliding back, whereas the neck screw remains impacted to the hole of the nail. In their prospective study of 56 patients with unstable intertrochanteric or subtrochanteric fractures, they had 3 cases with $Z$ effect and 2 with reverse Z-effect. The authors in an effort to prevent the Z-effect phenomenon suggest the use of a "ring" in the lateral side of the hip pin.In our study we had one case with reverse Zeffect (1.6\%)(Fig-4A).Simmermacher et al. (1999), in a clinical multicenter study, reported technical failures of the PFN after poor reduction, malrotation or wrong choice of screws in $5 \%$ of the cases ${ }^{10}$. A cut-out of the neck screw occurred in $0.6 \%$.In our study we had $6.6 \%$ failure rate with 2 cases of non union due to implant breakage and 2 cases of delayed union. We had a re-operation rate of $10 \%$ in our study due to implant breakage and mechanical problems like Z-effect and reverse Z-effect.

\section{Conclusion}

PFN is a viable option for treatment of subtrochanteric fractures with high union rate and minimal soft tissue damage. Intramedullary fixation has biological and biomechanical advantages, but the operation is technically demanding. Gradual learning and great patience is needed in order to make this method truly minimally invasive.

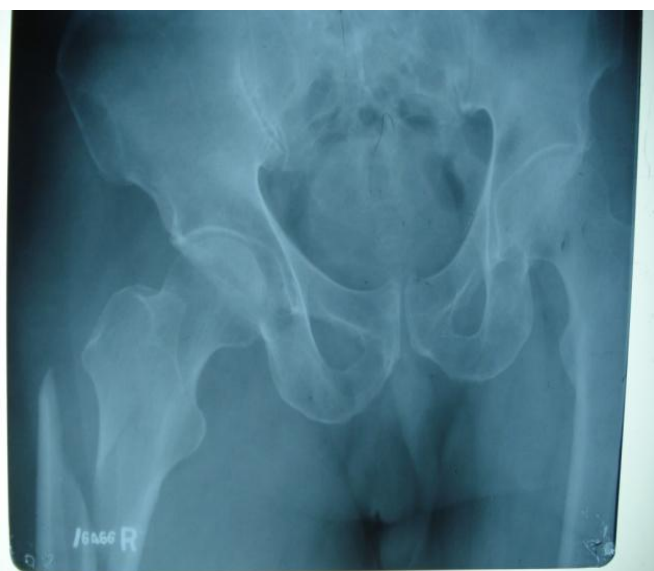

Figure 1A: Rays of 42 yrs old patient with right subtrochanteric fracture femur(Russel and Taylor Classification Type 1A) .

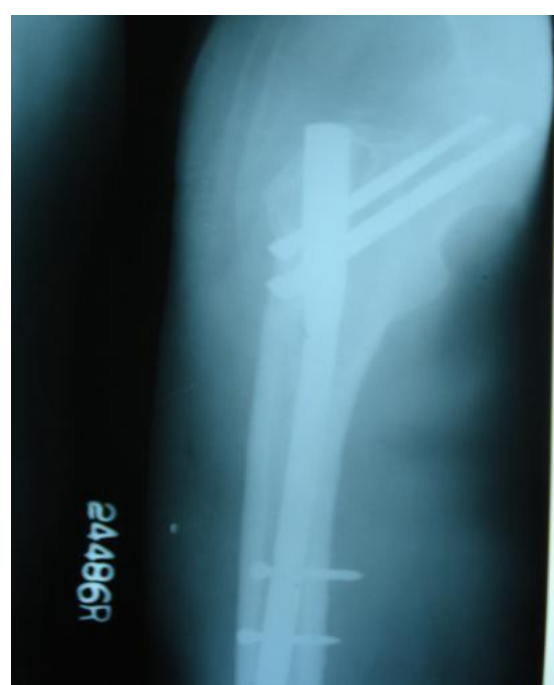

Figure 1B: X-Rays of same patient showing fracture union at 8 months after surgery.

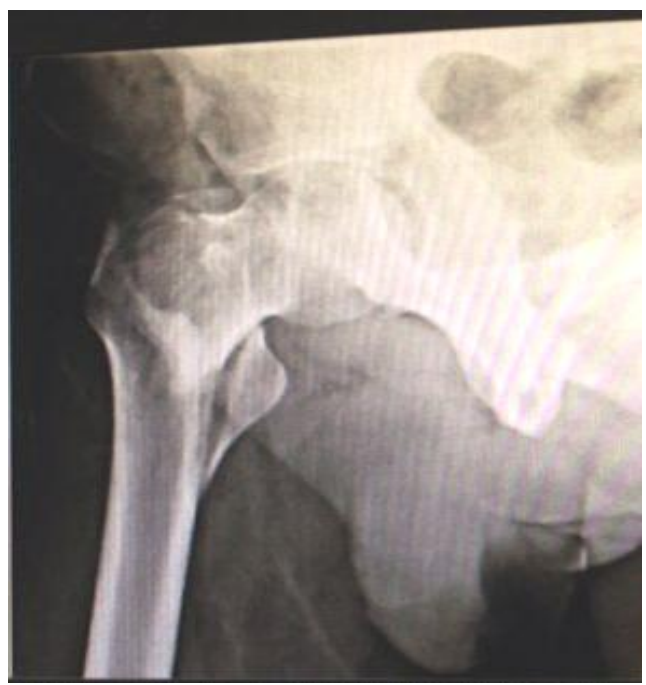

Figure 2A: X-Rays of 48 years old male patient with right subtrochanteric fracture femur(Russel and Taylor Classification Type 2A)

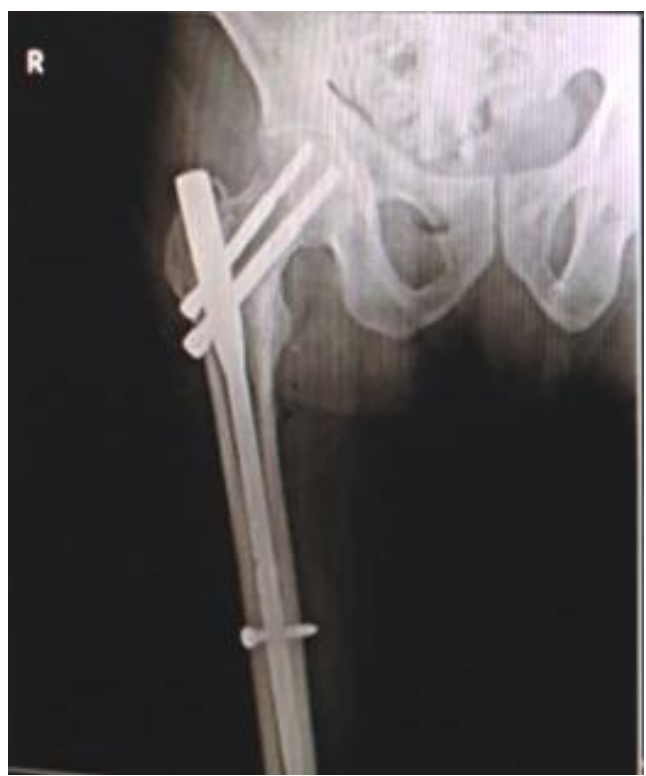

Figure 2B: X-Rays of the same patient showing fracture union at 10 months after surgery. 


\section{International Journal of Science and Research (IJSR) \\ ISSN (Online): 2319-7064}

Index Copernicus Value (2013): 6.14 | Impact Factor (2014): 5.611

\section{Complications}

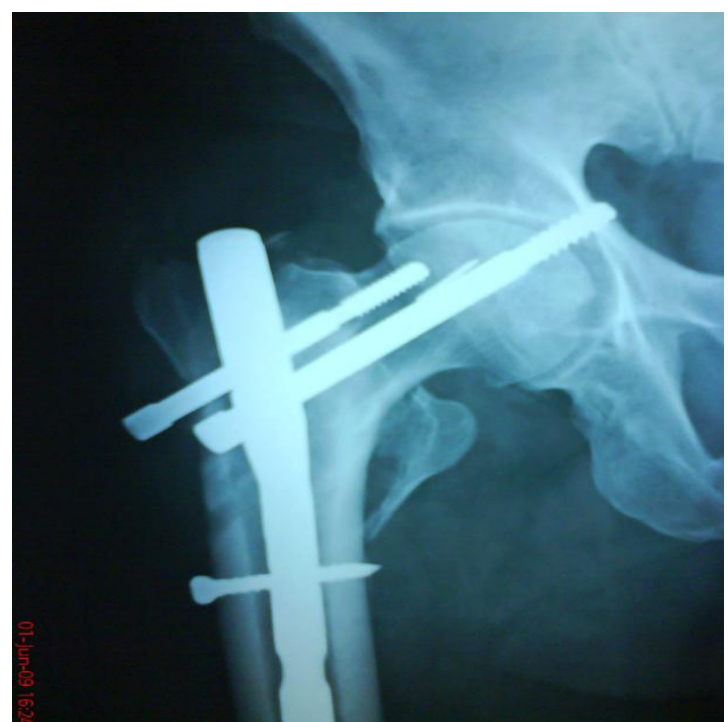

Figure 3A: X-Rays of a patient showing Z-effect.

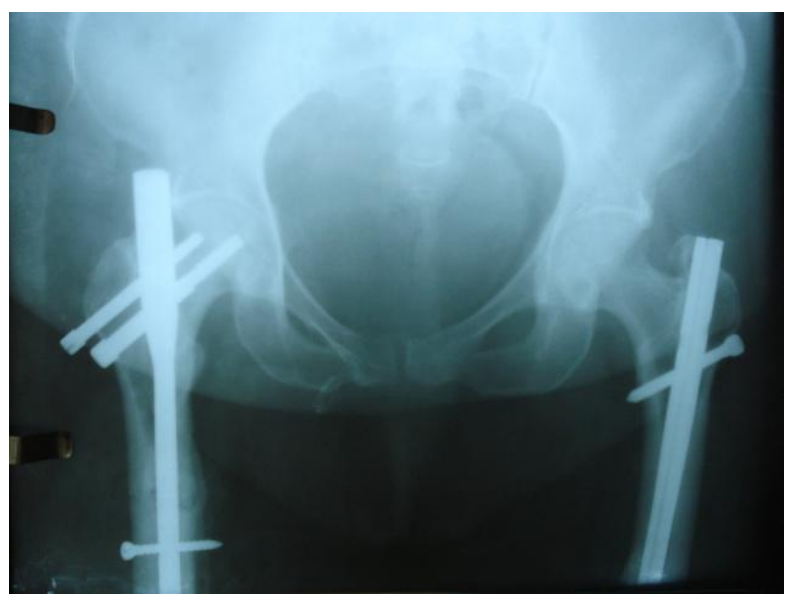

Figure 4A: X-Rays of a patient showing reverse Z-effect.

\section{References}

[1] Bucholz, Robert W, Heckman, James D, Court-Brown, Charles M. Rockwood and Green's Fractures in Adults, $6^{\text {th }}$ Edition.Lippincott Williams And Wilkins 2006;2:46.

[2] D'Aubigne RMPostelM. Functional results of hip arthroplasty with acrylic prosthesis. J Bone Joint Surg Am 1954;35:451-475.

[3] Chakraborty MK, Thapa P. Fixation of a subtrochanteric fracture of the femur. J Clin Diagn Res 2012;5:76-80.

[4] LS Jiang, Sheng L, Dai LY, Ren JY. Intramedullary fixation of subtrochanteric fractures with long proximal femoral nail or long gamma nail:technical notes and preliminary results. Ann Acad Med Singapore 2007;36:821-826.

[5] Parker MJ, Dutta BK, Sivaji C, et al. Subtrochanteric fractures of the femur.Injury 1997; 28:91-95.

[6] DeLee JC, Clanton TO, Rockwood CA Jr. Closed treatment of subtrochanteric fractures of the femur in a modified cast-brace. J Bone Joint Surg Am 1981;63:773- 79 .
[7] Boriani S, De lure F, Betelli G, Specchia I,Bungaro P, Montanari G, et al. The results of multicentric Italian study on the use of the Gamma nail for the treatment of pertrochanteric and subtrochanteric fractures: a reveiw of 1181 cases.Chir Organi Mov 1994;79:193-203.

[8] Banan H, Al-Sabti A, Jimulia T, et al.The treayment of unstable, extracapsular hip fractures with $\mathrm{AO} / \mathrm{ASIF}$ proximal femoral nail(PFN)-our first 60 cases. Injury 2002;33(5):401405.

[9] Christian Boldin" The proximal femoral nail-a minimal invasive treatment of unstable proximal femoral fractures" Acta Orthop Scand2003:74(1); 53- 58.

[10] Simmermacher R K J, Bosch A M, Van der Werken C. The AO/ASIF- proximal femoral nail (PFN): a new device for the treatment of unstable proximal femoral fractures. Injury 1999; 30: 327-32. 